\title{
Sulforaphane-cysteine inhibited migration and invasion via enhancing mitophagosome fusion to lysosome in human glioblastoma cells
}

\author{
Yan Zhou', Yalin Wang ${ }^{1}$, Sai Wu', Yuting Yan', Yabin Hu' ${ }^{1}$, Zhongnan Zheng ${ }^{1}$, Juntao $\mathrm{Li}^{1}$ and Wei Wu ${ }^{1,2}$
}

\begin{abstract}
Here we uncovered the involved subcellular mechanisms that sulforaphane-cysteine (SFN-Cys) inhibited invasion in human glioblastoma (GBM). SFN-Cys significantly upregulated 45 and downregulated 14 microtubule-, mitophagy-, and invasion-associated proteins in GBM cells via HPLC-MS/MS and GEO ontology analysis; SFN-Cys disrupted microtubule by ERK1/2 phosphorylation-mediated downregulation of a-tubulin and Stathmin-1 leading to the inhibition of cell migration and invasion; SFN-Cys downregulated invasion-associated Claudin-5 and S100A4, and decreased the interaction of a-tubulin to Claudin-5. Knockdown of Claudin-5 and S100A4 significantly reduced the migration and invasion. Besides, SFN-Cys lowered the expressions of a-tubulin-mediated mitophagy-associated proteins Bnip3 and Nix. Transmission electron microscopy showed more membrane-deficient mitochondria and accumulated mitophagosomes in GBM cells, and mitochondria fusion might be downregulated because that SFN-Cys downregulated mitochondrial fusion protein OPA1. SFN-Cys increased the colocalization and interplay of LC3 to lysosomal membrane-associated protein LAMP1, aggravating the fusion of mitophagosome to lysosome. Nevertheless, SFN-Cys inhibited the lysosomal proteolytic capacity causing LC3II/LC3I elevation but autophagy substrate SQSTM1/ p62 was not changed, mitophagosome accumulation, and the inhibition of migration and invasion in GBM cells. These results will help us develop high-efficiency and low-toxicity anticancer drugs to inhibit migration and invasion in GBM.
\end{abstract}

\section{Introduction}

High invasion in human glioblastoma (GBM) is the key reason to cause low survival and bad outcome ${ }^{1}$. Plantderived sulforaphane (SFN) induced apoptosis and inhibited invasion by ERK1/2 phosphorylation in various cancers $^{2-5}$. Sulforaphane-cysteine (SFN-Cys), one of the SFN metabolites, has great potential to penetrate blood-brain barrier and a longer half-life in the brain tissue than $\mathrm{SFN}^{6}$. We previously reported that SFN-Cys inhibited migration and invasion via microtubulemediated Claudins dysfunction in human non-small cell

\footnotetext{
Correspondence: Wei Wu (weiwu207@ccmu.edu.cn)

'Department of Biochemistry and Molecular Biology, School of Basic Medical Sciences, Capital Medical University, Beijing 100069, China

${ }^{2}$ Beijing Key Laboratory for Invasion and Metastasis, Capital Medical University, No. 10, Xitoutiao, You An Men Wai Ave., Feng Tai District, Beijing 100069, China Edited by G. M. Fimia
}

lung cancer (NSCLC) cells ${ }^{7}$. We also revealed that SFNCys suppressed invasion via downregulating Galectin-1 in human prostate cancer ${ }^{8}$. Here at subcellular levels we will determine how SFN-Cys inhibited migration and invasion in human GBM to establish a high-efficiency anticancer chemotherapy.

Microtubule plays a vital role in cell motility, migration, and invasion of tumor cells via assembly and disassembly of $\alpha$-tubulin and $\beta$-tubulin complex ${ }^{9}$. Actins and tubulins form highly dynamic polymers that are capable of organizing cytoplasmic organelles, determining cell shape and polarity and promoting cell-cell and cell-matrix adhesions through their interactions with cadherins and integrins, respectively ${ }^{10}$. Studies showed that microtubule-associated protein Stathmin-1 binds to tubulins promoting the depolymerization of microtubule ${ }^{11}$, while the inhibition of stathmin expression significantly reduced transendothelial 
migration in neuroblastoma cells ${ }^{12}$. Just recently, we found that SFN-Cys triggered microtubule disruption via decreasing Stathmin-1 leading to microtubule depolymerization in human prostate cancer $^{13}$. Therefore, we predicted that SFN-Cys might inhibit migration and invasion via regulating Stathmin-1-assoicated microtubule disruption in human GBM cells.

Invasion-associated proteins, membrane proteins, or adhesion molecules might contribute to tumor migration and invasion. Claudin-5 is one of the main components of tight junctions for cancer cells to connect adjacent cells ${ }^{14}$. Studies demonstrated that cell proliferation and invasion were reduced after silencing Claudin $-5^{15}$. We previously reported that sustained phosphorylation of ERK1/2 contributed to invasion inhibition ${ }^{5,8}$. SFN promoted the phosphorylation of ERK1/2 downregulating matrix metalloproteinase MMP-2 leading to invasion inhibition in $\mathrm{GBM}^{4}$. Claudin-5 was determined to interact with the MT1 matrix metalloproteinase increasing cell migration and invasion via degradation of the extracellular matrix $^{16,17}$. We found that knockdown of $\alpha$-tubulin downregulated Claudins and inhibited migration and invasion, indicating that microtubule disruption contributed to invasive inhibition in NSCLC cells ${ }^{7}$. The cytosolic C-terminal domain of Claudin-5 contains a PDZ-binding domain which binds ZO-1, ZO-2, and ZO-3 proteins, consequently binding to the microtubules ${ }^{18}$. Besides, Claudin- 11 was proved to interact with $\alpha$-tubulin promoting cell migration, indicating that microtubule might function as a mediator to regulate Claudins signaling, autophagy and invasion ${ }^{19}$. SFN-Cys might downregulate a dozen of proteins via phosphorylated ERK1/2mediated signals ${ }^{7,8,13}$. S100A4 is a member of the S100 protein family, which is associated with cell motility ${ }^{20}$. S100A4 is known to promote invasion and metastasis in human GBM cells ${ }^{21}$; knockdown of S100A4 reduced tumorigenesis and metastasis in tumor cells ${ }^{22}$. Therefore, here it is necessary to investigate whether SFN-Cys inhibits invasion via regulating S100A4 and Claudin-5.

Autophagy is an intracellular degradation process maintaining cell homeostasis by clearing damaged organelles and proteins; microtubule played a critical role in regulating such a process. At autophagy, microtubuleassociated protein light chain 3 II (LC3II) was recruited onto autophagosomal membrane, moving along microtubule to fuse autophagosome to lysosome for degradation, and the level of autophagic substrate SQSTM1/p62 (sequestosome 1) decreased ${ }^{23,24}$. We previously reported that another SFN metabolite, sulforaphane-N-acetylcysteine (SFN-NAC) induced microtubule disruption promoting the formation and accumulation of autophagosome leading to apoptosis in NSCLC cells ${ }^{25}$. Besides, both SFN-Cys and SFN-NAC also inhibited migration and invasion via interfering autolysosome formation in
NSCLC cells ${ }^{13}$. However, at subcellular level, it is not clear how SFN and its metabolites regulate mitophagy inhibiting cell migration and invasion.

Mitophagy maintains cell homeostasis via specifically degrading abnormal mitochondria. Both Bnip3 (Bcl-2/ adenovirus E1B 19-kDa-interacting protein 3) and Nix (BNIP3L) interact directly with LC3 to promote mitophagy $^{26}$. Bnip3 is a mitochondrial BH3-only protein that contributes to mitophagy ${ }^{26}$. Its receptor Nix directly interacts with LC3, and mediated the subsequent binding and sequestration of mitochondrial proteins to autophagosomes $^{25}$. Besides, Bnip3 increases the rate of mitophagy in response to hypoxia promoting tumor progression to metastasis ${ }^{27}$. In the present study, we will investigate whether SFN-Cys regulates mitochondrial autophagy by changing microtubule dynamics, leading to the inhibition of migration and invasion in GBM.

Taken together, SFN-Cys might cause microtubule disruption, regulate mitophagy and the expression of S100A4 and Claudin-5 inhibiting migration and invasion in GBM cells; the investigation of the underlying mechanisms will help us design brand-new, effective and safe drugs to treat invasive cancers.

\section{Materials and methods \\ Reagents and antibodies}

D, L-sulforaphane-L-cysteine (SFN-Cys) (sc-207499) was purchased from Santa Cruz Biotechnology (Dallas, Texas, USA). Anti-p-ERK1/2 (9101S), anti-ERK1/2 (9102S), antiLAMP1 (15665), and PD98059 (9900S) were obtained from Cell Signaling Technology (Danvers, MA, USA). AntiStathmin-1 (D260545) and anti-pStathmin-1 (Ser 25) (D155102) were purchased from Sangon Biotech (Shanghai, China). Anti-Claudin-5 (ab131259), anti-S100A4 (ab124805), anti-Bnip3 (ab109362), anti-Nix (ab109414), anti- antiSQSTM1/p62 (ab207305), and anti-OPA1 (ab157457) were purchased from Abcam (Cambridge, UK). Matrigel basement membrane matrix (356234) was bought from BD Biosciences (Franklin Lakes, NJ, USA). Mitochondrial Isolation Kit (ProteinExt ${ }^{\mathrm{TM}}$ ) (DE401-01) was purchased from TransGen Biotech (Beijing, China). Mitophagy inducer carbonyl cyanide 3-chlorophenylhydrazone (CCCP) (C2759) was purchased from Sigma-Aldrich (St-Louis, Missouri, USA). Bafilomycin A1 (Baf-A1) (S1413) was purchased from Selleck Chemicals (Houston, DX, USA). Chloroquine diphosphate (T0194) was purchased from Topscience (Shanghai, China). Anti- $\alpha$-tubulin (11224-1-AP) and anti$\beta$-actin (60008-1-Ig) were ordered from Proteintech (Wuhan, Hubei, China).

\section{Cell culture}

Human GBM U87MG cell line was purchased from the Cell Resource Center, Peking Union Medical College (CRC/PUMC) and U373MG cell line was purchased from 
American Type Culture Collection (ATCC, USA). The species origin was confirmed with PCR, and the cell identity was authenticated with STR profiling by FuHeng Biology (Shanghai, China). Cells were cultured in DMEM/ HIGH glucose culture medium (SH30243.01B, Hyclone, Logan, USA) supplemented with $10 \%$ fetal bovine serum (FBS) (SE100-B, Vistech, Australia), $100 \mathrm{U} / \mathrm{ml}$ penicillin and $100 \mathrm{U} / \mathrm{ml}$ streptomycin solution (P1400-100) (Solarbio, Beijing, China) in a standard humidified incubator with $5 \% \mathrm{CO}_{2}$ at $37^{\circ} \mathrm{C}$. Generally, the cultured cells in logarithmic phase were randomly allocated to group and treated by SFN-Cys and/or other stimuli in each assay.

\section{High performance liquid chromatography-mass spectrometry/mass spectrometry (HPLC-MS/MS)}

For the glycopeptide identification, HPLC-MS/MS analysis was performed via an Orbitrap Fusion Lumos mass spectrometer (Thermo Scientific, USA) equipped with a nanoelectrospray ionization source and an EASYnLC 1000 liquid chromatography system (Thermo Scientific, USA). The samples were dissolved in $0.1 \% \mathrm{FA}$ and separated on a capillary column $(150 \mu \mathrm{m}$ id $\times 120 \mathrm{~mm})$ packed with $\mathrm{C} 18(1.9 \mu \mathrm{m}, 100 \AA$,) at a flow rate of $600 \mathrm{nl} /$ min. The mobile phase consisted of $0.1 \%$ formic acid in water (A) and $0.1 \%$ formic acid in acetonitrile (ACN) (B). Mobile phase A ( $99.9 \%$ water/0.1\% FA) and mobile phase B (99.9\% ACN/0.1\% FA) were used, and the elution gradient used was from 6 to $32 \%$ mobile phase B for $78 \mathrm{~min}$. Data acquisition was performed by the data-dependent mode. The recognized peptides were searched from UniProt Knowledgebase (https://www.uniprot.org/). The threshold of 1.5 -fold or 0.66 -fold changes $(p<0.05)$ was defined based on the ratio of values in the treated group versus control group as an upregulation or downregulation. The control or SFN-Cys-treated group was performed in triplicate.

\section{Western blot}

Total cellular protein lysates were harvested and lysed in RIPA lysis buffer (C1053, Applygen, Beijing, China) with a protease inhibitor cocktail (04693132001, Roche, Shanghai, China). Protein concentration was evaluated by the BCA protein assay kit (P1511, Applygen, Bejing, China). Equal amounts of total proteins were separated with SDS-PAGE (12\%) for the detection of ERK1/2, pERK1/2, Stathmin-1, pStathmin-1, $\alpha$-tubulin, Claudin-5, S100A4, Bnip3, Nix, SQSTM1/p62, and OPA1. $\beta$-actin was used as the protein loading control. After SDS-PAGE, the proteins in the gels were transferred to BioTrace nitrocellulose membranes (66485, Pall, New York, USA) with $7.5 \%$ bovine serum albumin (BSA) blockage in Trisbuffered saline (pH7.4, $20 \mathrm{mM}$ Tris- $\mathrm{HCl}, 150 \mathrm{mM} \mathrm{NaCl}$ ), and then incubated with afore-mentioned primary antibodies overnight at $4{ }^{\circ} \mathrm{C}$. The membranes were incubated with IRDye-labeled goat anti-mouse or goat antirabbit IgG (LI-COR, Lincoln, NE, USA) at the room temperature for $1 \mathrm{~h}$. Finally, the protein bands were scanned by the LI-COR Odyssey system (LI-COR, Lincoln, NE, USA). At least three independent experiments were performed. The grayscale of the protein bands was calculated and normalized by densitometry of $\beta$-actin signal by Image J software.

\section{RNA interference}

Claudin-5 siRNA (5'-UUCAUUCCGUCUGUUAAGG TT $\left.-3^{\prime}\right)^{28}$ and designed S100A4 siRNA (5'-UACUUGUGG AAGGUGACACCAUTT-3') as well as control siRNA (5'-ACGUGACACGUUCGGAGAATT-3') were synthesized to silence Claudin-5 and S100A4 (Gene Pharma, Shanghai, China). Cells were plated in six-well plates and cultured for $24 \mathrm{~h}$. Then the siRNAs $(30 \mathrm{pmol} /$ well) with Lipofectamine $^{\text {TM }}$ RNAiMAX (13778075, Invitrogen, CA, USA) were transfected into cells when cells reached $\sim 80 \%$ confluency. After the transfected cells were cultured for more than $24 \mathrm{~h}$, cells were randomly divided into two plates and treated with or without SFN-Cys at 60-70\% confluency.

\section{Scratch assay}

Wound scratch healing assay was performed to detect the capacity of cell migration. Cells were seeded at a density of $2 \times 10^{6}$ cells per well in a six-well plate and cultured overnight. Three parallel thin "wounds" and one vertical "wound" were scratched by a pipette tip when cells reach $\sim 100 \%$ confluency. The cells were washed with phosphate-buffered saline (PBS) and treated by serial concentrations of SFN-Cys with serum-free medium for $24 \mathrm{~h}$. The images were captured by a phase-contrast microscope at 0 and $24 \mathrm{~h}$, and the relative wound areas were calculated by the Image-pro plus 6.0. The migrated cells were observed in at least five to six randomly selected fields per well under microscope. The ratio of wound area in $0 \mathrm{~h}$ vs. $24 \mathrm{~h}$ represented the inhibitory capacity of migration and invasion by SFN-Cys. At least three independent experiments were performed.

\section{Invasion assay}

The transwell chambers placed into a 24-well plate were rehydrated at $37^{\circ} \mathrm{C}$ for $1 \mathrm{~h}$ before seeding cells. Matrigel basement membrane matrix was diluted with serum-free DMEM medium to $2 \mathrm{mg} / \mathrm{ml}$ and then plated onto transwell chamber. A total of $2 \times 10^{4}$ cells were added to the upper chamber with serum-free DMEM medium and $500 \mu \mathrm{l}$ DMEM medium containing 10\% FBS was added to the lower chamber. After treated with different concentrations of SFN-Cys for $24 \mathrm{~h}$, the cells were fixed with $100 \%$ methanol for $20 \mathrm{~min}$ and then stained with $0.5 \%$ 
crystal violet solution for $30 \mathrm{~min}$. The cells were washed three times with PBS and the cells on the top of upper chamber were removed gently with a cotton swab. The invasive cells were observed in at least five to six randomly selected fields per well under microscope. The data were analyzed by Image J software. At least three independent experiments were performed.

\section{Bioinformatics analysis}

We searched the GEPIA (Gene Expression Profiling Interactive Analysis) Database to find the possible correlations between survival rate and expression to microtubule related proteins. GEPIA is a public database newly developed by the Chinese for cancer and normal gene expression profiling from 9736 tumors and 8587 normal samples. These results with statistical significance will be recorded $(p<0.05)^{29}$. We also searched the version 10.5 of STRING database to get the data for protein correlation and drew the Protein-Protein Interaction Map using Cytoscape software ${ }^{30}$.

\section{Immunofluorescence staining and confocal microscopy}

Cells were seeded in $35 \mathrm{~mm}$ cover glass-bottom dishes at a density of $1 \times 10^{5}$ cells/dish and incubated for $24 \mathrm{~h}$, then treated with $20 \mu \mathrm{M}$ SFN-Cys and/or $10 \mu \mathrm{M}$ CCCP. The cells were fixed with $4 \%$ paraformaldehyde for $15 \mathrm{~min}$. After washed by PBST (PBS with Tween-20), the cells were permeabilized with $-20^{\circ} \mathrm{C}$ methanol for $10 \mathrm{~min}$ at the room temperature. After blocking with PBS containing $1 \%$ BSA and $0.1 \%$ Triton $\mathrm{X}-100$ for $1 \mathrm{~h}$, the cells were incubated with anti- $\alpha$-tubulin or anti-LAMP1 and anti-LC3 overnight at $4{ }^{\circ} \mathrm{C}$. After washed three times with PBST, the cells were incubated with the fluorescencelabeled secondary antibody for $1 \mathrm{~h}$ at the room temperature, followed with adding fluorescent mounting medium with DAPI (4,6-diamidino-2-phenylindole) (DAPI) (ZLI9557, ZSGB-Bio, China). All the measurements were performed by a researcher who was blinded to group allocation with a laser scanning confocal microscope (Olympus FV1000, Olympus, Japan). The Pearson's_Rr was calculated by Image J software.

\section{Co-immunoprecipitation}

Cells were plated at a density of $5 \times 10^{6}$ cells/dish and treated with $20 \mu \mathrm{M}$ SFN-Cys for $24 \mathrm{~h}$. After washed with ice-cold PBS, cells were lysed on ice via Nondenaturing Lysis Buffer (C1050, Applygen, Beijing, China) with protease inhibitor cocktail (04693132001, Roche, Shanghai, China). After total proteins were quantified, equal amount of proteins were incubated with the fixed primary antibodies overnight at $4{ }^{\circ} \mathrm{C}$. Then, the complexes were coincubated with protein A/G agarose (sc-2003, SantaCruz, Dallas, Texas, USA) rotationally for $3 \mathrm{~h}$ at $4{ }^{\circ} \mathrm{C}$ and the proteins were isolated from the beads by centrifuging and boiled for $5 \mathrm{~min}$. Western blot was used to recognize the conjugated proteins.

\section{Transmission electron microscopy}

Cells were incubated with or without $20 \mu \mathrm{M}$ SFN-Cys for $24 \mathrm{~h}$, then harvested and fixed with $3 \%$ glutaraldehyde at $4{ }^{\circ} \mathrm{C}$ for $2 \mathrm{~h}$. After washed with PBS for three times, samples were fixed in $1 \%$ osmium tetroxide for $1 \mathrm{~h}$. Then samples were dehydrated through a graded ethanol series and embedded in a 1:1 mixture of acetone and Epon812 resin for $30 \mathrm{~min}$. The samples were then infiltrated $2 \mathrm{~h}$ in Epon812. Ultrathin sections were cut with a knife and placed on 200-mesh copper grids and stained with Uranium acetate for $30 \mathrm{~min}$ and Lead nitrate for $20 \mathrm{~min}$. All the measurements were performed by a researcher who was blinded to group allocation with a transmission electron microscope (JEM-1400Plus, JEOL, Japan).

\section{Mitochondrial protein extraction}

Cells $\left(2 \times 10^{7}\right)$ were treated with or without $20 \mu \mathrm{M}$ SFNCys for $24 \mathrm{~h}$, then were harvested by centrifugation at $1000 \times g$ for $3 \mathrm{~min}$. Mitochondrial Isolation Kit (Protei$\mathrm{nExt}^{\mathrm{TM}}$ ) (DE401-01, TransGen, Beijing, China) was used to isolate mitochondrial proteins by the manufacturer's instructions. Cells were lysed with $400 \mu \mathrm{l}$ MIB I and $5 \mu \mathrm{l}$ MIB II, respectively, for 2 and $5 \mathrm{~min}$ on ice with severe shake. After mixed with $400 \mu \mathrm{l}$ MIB III, cells were centrifuged at $700 \times g$ at $4{ }^{\circ} \mathrm{C}$ for $10 \mathrm{~min}$. The supernatant was centrifuged again at $12,000 \times g$ at $4{ }^{\circ} \mathrm{C}$ for $15 \mathrm{~min}$; the harvested supernatant contained the cytosolic proteins. Then the obtained pellet was suspended with $500 \mu \mathrm{l} \mathrm{MIB}$ III and was centrifuged at $12,000 \times g$ at $4{ }^{\circ} \mathrm{C}$ for $15 \mathrm{~min}$. The final pellet was lysed to prepare the mitochondrial proteins.

\section{Autophagy and mitophagy assay}

Western blot analysis was used to detect the LC3II, the indicator of autophagosome formation. Mitophagy inducer CCCP $(10 \mu \mathrm{M})$ was used to induce mitophagy to test the migration and invasion in tumor cells. Bafilomycin A1 (Baf$\mathrm{A} 1,50 \mathrm{nM})$ or chloroquine $(25 \mu \mathrm{M})$ was used to block the fusion of autophagosome/mitophagosome to lysosome and inhibit the lysosomal activity, respectively. TEM was performed to observe the subcellular morphology.

\section{Statistical analysis}

All data represent at least three independent experiments. Differences among groups and treatments for all in vitro experiments were determined by two-tailed Student's $t$ test in GraphPad Prism and Excel. Bar graph represents quantification data as means \pm SD from at least three biological replicates $(n \geq 3)$. $P$ values of less than 0.05 represented statistical significance. 


\section{Results}

SFN-Cys regulated proteomic expressions and inhibited migration and invasion in a dose-dependent manner

SFN-Cys upregulated 45 and downregulated 14 protein expressions in U87MG cells via HPLC-MS/MS analysis; these proteins were associated with microtubule, mitophagy, migration, and invasion (Fig. 1a) (Supplementary Table 1). Of these, gene expression analysis by GEPIA server showed that the expressions of TUBA1C, S100A4, CLDN5 (Claudin-5), MAPK1, LAMP1, LAMP2, BNIP3L (Nix), MAP1LC3B (LC3), PODXL, MMP-2, ITGB3, CD47, and FN1 were upregulated, while SEMA7A expression was downregulated in GBM tissues (Fig. 1b). Protein-Protein Interaction network was done and the results showed that both CLDN5 and S100A4 might interact with invasion-associated matrix metalloproteinase MMPs (Fig. 1c). Besides, TUBA1C, STMN1, BNIP3, BNIP3L, MAP1LC3B, LAMP1, LAMP2, MAPK1, and MAPK3 might interact one another (Fig. 1c). Survival analysis showed that the expressions of S100A4, FN1, and MMP-2 were negatively correlated to over-survival significantly in GBM and Brain Lower Grade Glioma (Fig. 1d). Further, the data obtained from Gene Correlation Analysis showed that the expression of TUBA1C was positively correlated to the expression of CLDN5 and S100A4; MAP1LC3B expression was positively correlated to the expressions of Bnip3, Nix and LAMP1; LAMP1 expression was positively correlated to S100A4 expression in GBM (Fig. 1e). We found that $20 \mu \mathrm{M}$ SFN-Cys inhibited migration and invasion without inducing apoptosis in U373MG and U87MG cells; this is the optimal dose for cell motility assay. It was shown that the number of migrant cells was significantly decreased with the increase of SFN-Cys dose by migration assay (Fig. 1f). Similarly, the number of invasive cells was significantly decreased with the increase of SFN-Cys dose by invasion assay (Fig. 1g). Also, the relative wound closures ( $0 \mathrm{~h}$ vs. $24 \mathrm{~h}$ ) were decreased with the increase of SFN-Cys dose by scratch assay (Fig. 1h). These indicated that SFN-Cys inhibited migration and invasion in U373MG and U87MG cells in a dose-dependent manner.

\section{SFN-Cys upregulated phosphorylated ERK1/2 inhibiting migration and invasion}

We have uncovered that SFN-Cys inhibited invasion in a couple of cancer types including NSCLC and prostate cancer $^{5,8}$. Here, results showed that SFN-Cys induced the phosphorylation of ERK1/2 (Thr202/Tyr204) in a dosedependent manner (Fig. 2a). However, phosphorylated ERK1/2 inhibitor PD98059 reversed the SFN-Cys-induced phosphorylation of ERK1/2 (Fig. 2b). Then, PD98059 was used to determine the linkage of SFN-Cys-regulated ERK1/ 2 signaling to cell migration and invasion in GBM cells. Results showed that the relative wound closures $(0 \mathrm{~h}$ vs.
$24 \mathrm{~h})$ were decreased after cells were treated with $20 \mu \mathrm{M}$ SFN-Cys by scratch assay, while such reduction was reversed by PD98059 (Fig. 2c). The numbers of migrant cells were significantly decreased after cells were treated with $20 \mu \mathrm{M}$ SFN-Cys by migration assay, while such reduction was reversed by PD98059 (Fig. 2d). Likewise, the invasive cells were decreased with the treatment of $20 \mu \mathrm{M}$ SFN-Cys by invasion assay in matrigel, which was reversed by PD98059 (Fig. 2e). These results indicated that SFN-Cys mediated the phosphorylation of ERK1/2 inhibiting the migration and invasion in human GBM cells.

\section{SFN-Cys-mediated ERK1/2 phosphorylation downregulated a-tubulin and Stathmin-1, upregulated phosphorylated Stathmin-1 triggering microtubule disruption}

We reported that SFN-Cys downregulated $\alpha$-tubulin in the previous studies ${ }^{13}$. Results showed that SFN-Cys downregulated $\alpha$-tubulin in a dose-dependent manner in GBM cells (Fig. 3a), while such downregulation was reversed by PD98059 $(25 \mu \mathrm{M})$ (Fig. 3b), indicating that SFN-Cys downregulated $\alpha$-tubulin by the phosphorylation of ERK1/2. Besides, SFN-Cys downregulated Stathmin-1 and upregulated pStathmin-1 (Ser 25), and these results were reversed by PD98059 (Fig. 3c, d). These data indicated that SFN-Cys modulated Stathmin-1 and pStathmin1 (Ser 25) via ERK1/2 phosphorylation in GBM cells. Meanwhile, we observed the morphological changes of microtubule by immunofluorescence assay. The results showed that microtubules were filamentous and distributed homogeneously in the control cells, while the cell tentacles disappeared and the microtubules were disrupted and aggregated in SFN-Cys-treated cells (Fig. 3e).

\section{SFN-Cys inhibited migration and invasion by downregulating Claudin-5 and S100A4}

It was shown that Claudin-5 and S100A4 were downregulated after cells were treated with $0,10,20,30 \mu \mathrm{M}$ SFNCys for $24 \mathrm{~h}$ by western blot in GBM cells (Fig. 4a, b). Claudin-5 and S100A4 siRNAs were successfully used to silence Claudin-5 and S100A4 (Fig. 4c, d). By scratch assay, SFN-Cys-induced decrease of migratory capacity was significantly aggravated by either Claudin-5 or S100A4 silencing (Fig. 4e, f). Further, invasion assay showed that SFN-Cysinduced decrease of invasion capacity was also significantly exacerbated by either Claudin-5 or S100A4 silencing (Fig. 4g, h). These results indicated that SFN-Cys inhibited migration and invasion via downregulating Claudin-5 and S100A4 in human GBM cells. Immunofluorescence and confocal microscopy results showed that SFN-Cys suppressed the colocalization of Claudin-5 to $\alpha$-tubulin (Fig. 4i). Meanwhile, by co-immunoprecipitation we found that SFN-Cys reduced the interaction of Claudin-5 to $\alpha$-tubulin (Fig. 4j). Therefore, SFN-Cys might reduce the microtubule-associated Claudin- 5 


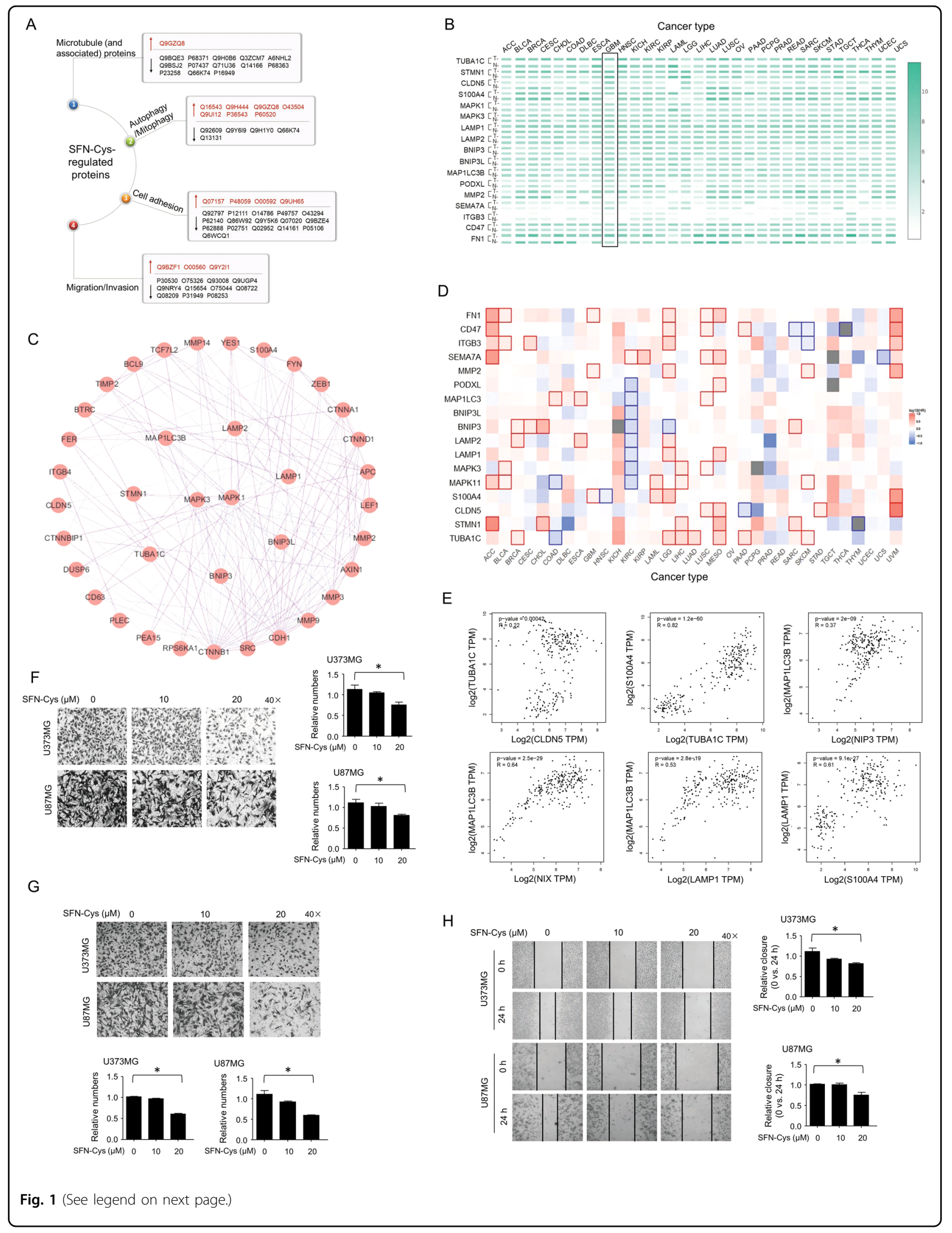


(see figure on previous page)

Fig. 1 SFN-Cys regulated proteomic expression and inhibited cell migration and invasion in U373MG and U87MG cells. a The SFN-Cysregulated protein expressions in U87MG cells were identified by HPLC-MS/MS. Red, upregulation; black, downregulation. b Gene Expression Profile was performed in predicted SFN-Cys-targeted proteins in either tumor (T) or normal ( $N$ ) tissue among different cancers by GEPIA. c The Protein-Protein Interaction network in SFN-Cys-targeted proteins was predicted by String server. $\mathbf{d}$ The Overall Survival Map of predicted SFN-Cystargeted proteins in different cancers by GEPIA. e The Correlation Analysis among SFN-Cys-targeted TUBA1C, CLDN5, S100A4, MAP1LC3B, Bnip3, Nix, and LAMP1 in GBM by GEPIA server $(p<0.05)$. Migration assay without matrigel (f) or invasion assay with matrigel $(\mathbf{g})$ was performed separately to detect the numbers of migratory or invasive cells in U87MG and U373MG cells after cells were treated with 0, 10, and $20 \mu \mathrm{M} \mathrm{SFN-Cys} \mathrm{for} 24 \mathrm{~h}$. $\mathbf{h}$ The area covered by migratory cells was recorded by Leica DMIRB microscope at $\times 40$ magnification at 0 and $24 \mathrm{~h}$. The relative closure $(0 \mathrm{~h}$ vs. $24 \mathrm{~h})$ was measured by Image J. At least three independent experiments were performed. ${ }^{*} p<0.05$. The values are expressed as means \pm SD $(n \geq 3)$.

causing the inhibition of migration and invasion in human GBM cells.

\section{SFN-Cys caused mitophagosome accumulation by disrupting microtubules}

Bnip3 and Nix are the mitophagy receptors that interact with LC3 promoting the sequestration of mitochondria into the isolation membrane. Here, we found that Bnip3 only presented in mitochondria (data not shown), and SFN-Cys decreased the expression of mitochondrial Bnip3 in a dose-dependent manner (Fig. 5a). Nevertheless, Nix was expressed both in mitochondria and cytoplasm, and SFN-Cys also downregulated both mitochondrial and cytosolic Nix in a dose-dependent manner (Fig. 5b, c). The expression of both Bnip3 and Nix were downregulated after knockdown of $\alpha$-tubulin via RNA interference (Fig. $5 \mathrm{~d}, \mathrm{e})$. These data indicated that SFN-Cys decreased $\alpha-$ tubulin causing the downregulation of Bnip3 and Nix, possibly inhibiting the formation of mitochondrial autophagosomes and selective clearance of mitochondria. However, SFN-Cys elevated the ratio of LC3II and LC3I (LC3II/LC3I) in a dose-dependent manner in both U373MG and U87MG cells (Fig. 5f), while unaffected the level of SQSTM1/p62 (Fig. 5g). SQSTM1/p62 is an autophagy substrate, and the reduction of SQSTM1/p62 was a marker of autophagy activation. Generally, SQSTM1/ p62 is degraded in a normal autophagic process; otherwise the level of SQSTM1/p62 is not changed. These results implied that SFN-Cys might block the autophagic flux and inhibit the degradation of autophagosomes. Besides, SFNCys-mediated upregulation of LC3II/LC3I was aggravated after $\alpha$-tubulin was silenced (Fig. $5 \mathrm{~h}$ ), suggesting that SFNCys decreased $\alpha$-tubulin level triggering the accumulation of autophagosomes. Bafilomycin A1 (Baf-A1) is a vacuole $\mathrm{H}^{+}$-ATPase inhibitor to inhibit the fusion of autophagosome to lysosome. Results showed that Baf-A1 elevated LC3II/LC3I, but Baf-A1-induced accumulation of LC3II was not further increased in the presence of SFN-Cys (Fig. 5i), indicating that SFN-Cys-mediated elevation of LC3II did not result from the formation of autophagosomes. $\mathrm{CCCP}$ is an uncoupling agent of mitochondrial proton carrier to destroy the mitochondrial membrane potential and induces mitophagy. TEM results showed that CCCP induced mitochondria disruption (Fig. 5j), while SFN-Cys induced more ruptured mitochondria and accumulated mitophagosomes coupled with apoptotic features in CCCP-treated cells than in sham group (Fig. 5j). Additionally, mitochondrial fusion protein Optic Atrophy 1 (OPA1) was significantly downregulated by SFN-Cys, while mitochondrial fission protein dynamin-related protein 1 was not affected (data not shown), suggesting SFN-Cys might inhibit mitochondrial fusion promoting mitochondrial fission, causing mitophagy inhibition (Fig. 5k). Likewise, SFN-Cys promoted the interaction of LC3II to lysosomal membrane-associated protein LAMP1 both in cytosol and mitochondria by co-immunoprecipitation (Fig. 5l). Further, we found that CCCP induced mitophagy leading to the elevated colocalization of LC3 to LAMP1 by confocal microscopy, while SFN-Cys exacerbated the colocalization of LC3 to LAMP1 in CCCP-treated cells, suggesting that SFN-Cys promoted the fusion of autophagosome to lysosome, which might be a cellular feedback against SFN-Cys stimulation (Fig. $5 \mathrm{~m}$ ). Chloroquine is a lysosomotropic agent. It accumulates inside the lysosomes causing inhibition of lysosomal enzymes that require an acidic $\mathrm{pH}$ and lysosomal protein degradation. Here, chloroquine significantly raised the conversion level of LC3I to II, while SFN-Cys did not further increase the conversion in chloroquine-treated cells (Fig. 5n), further suggesting that SFN-Cys impeded the autophagic flux and the degradation of autophagosome. However, we found that SFN-Cys downregulated lysosome-associated proteins or lysosomal proteases in the whole cells, such as Cathepsin $\mathrm{D}$ and tripeptidyl-peptidase 1 (TPP1), and downregulated Cathepsin $1 \mathrm{~L}$ in mitochondria by HPLC-MS/MS (Supplementary Table 2). Therefore, based on the results above, we concluded that SFN-Cys promoted the fusion of autophagosome to lysosome, but lowered the lysosomal degradation capacity, resulting in mitophagosome accumulation and mitophagy dysfunction.

\section{SFN-Cys inhibited migration and invasion by inhibiting mitophagy}

Here we found that the expression of Claudin-5 and S100A4 were downregulated in Baf-A1-treated cells, and SFN-Cys exacerbated the downregulation in Baf-A1 


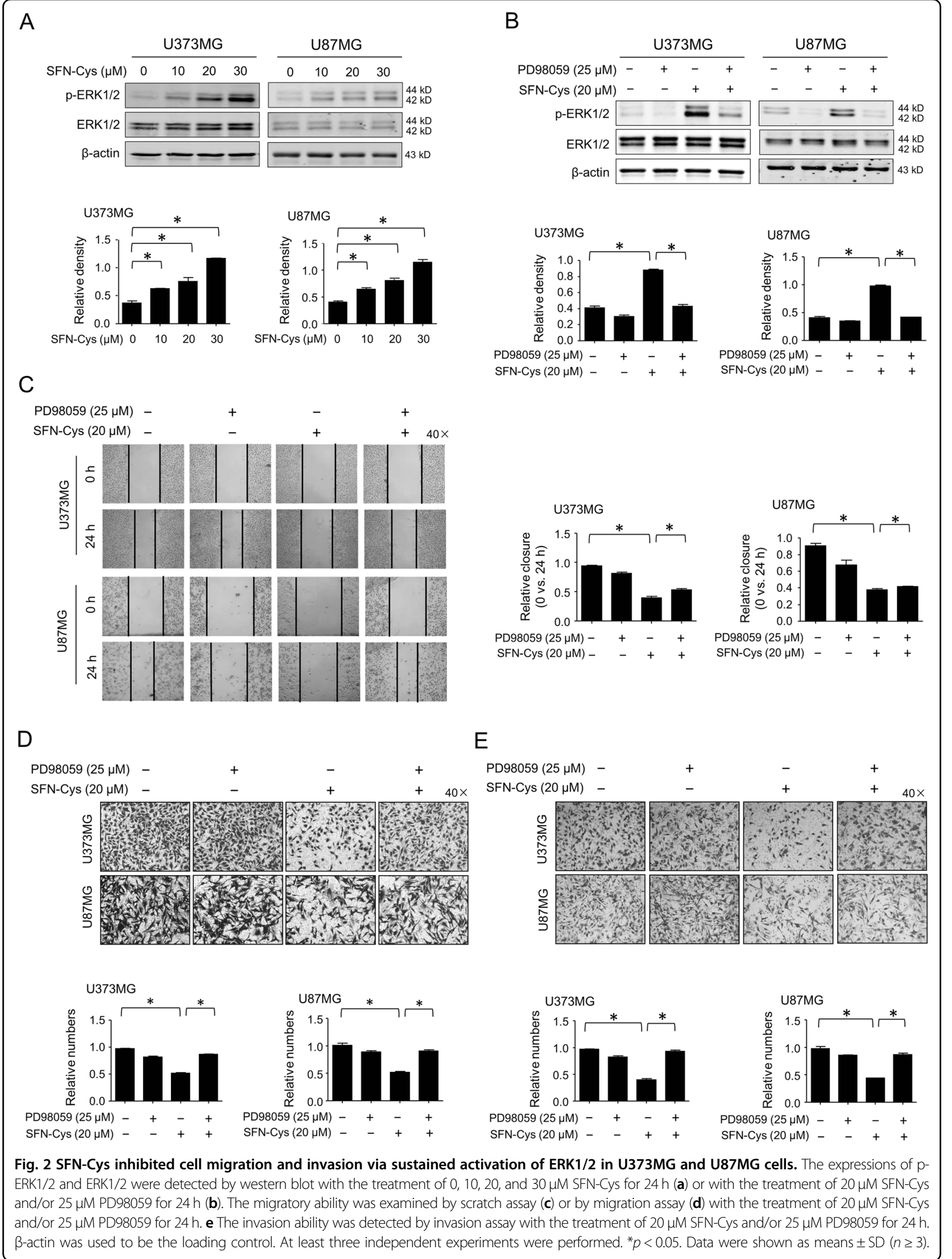


A
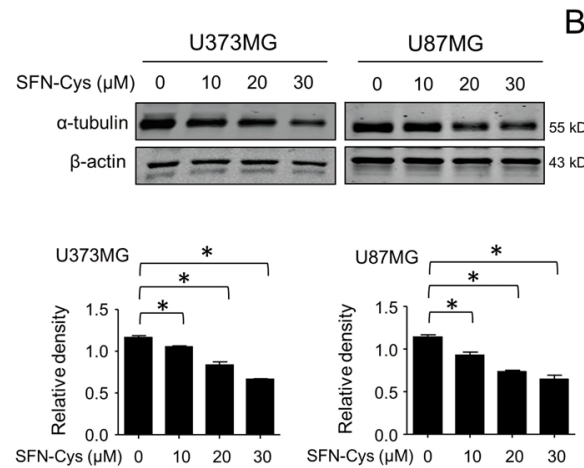

SFN-Cys $(\mu \mathrm{M}) \quad 0 \quad 10 \quad 20 \quad 30$

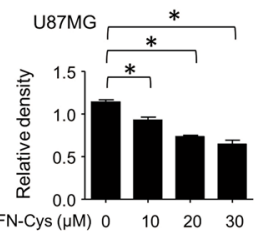

C
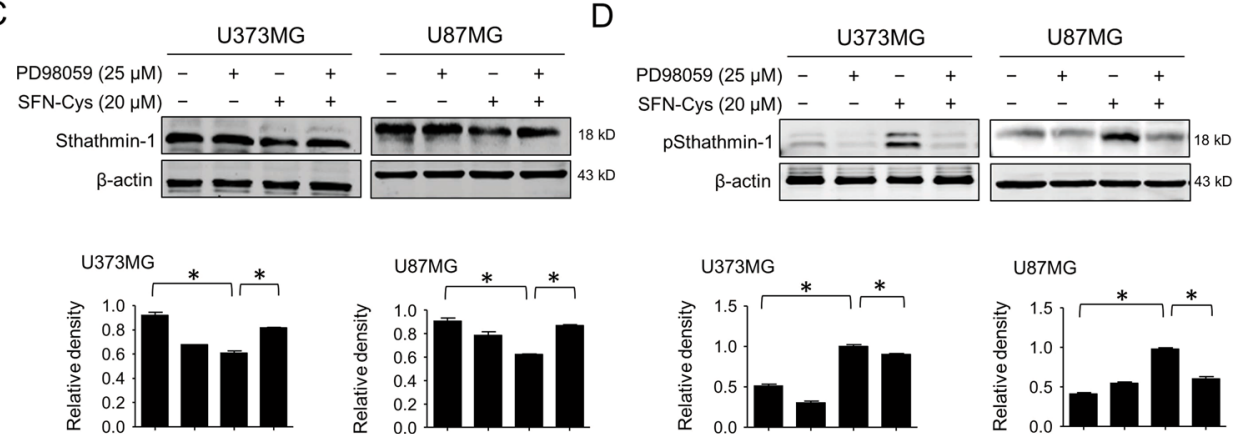
$\mathrm{PD} 98059(25 \mu \mathrm{M})$ SFN-Cys $(20 \mu \mathrm{M})$ -

B
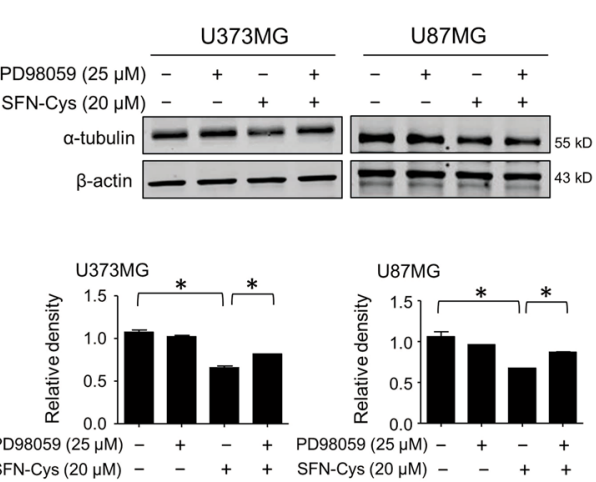

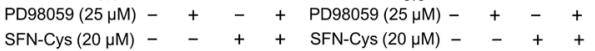

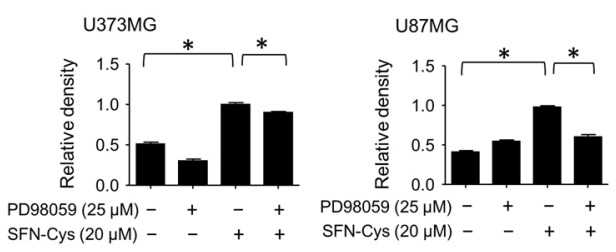

SFN-Cys $(20 \mu \mathrm{M})-+\quad+\quad+$ SFN-Cys $(20 \mu \mathrm{M})-\quad++$

E
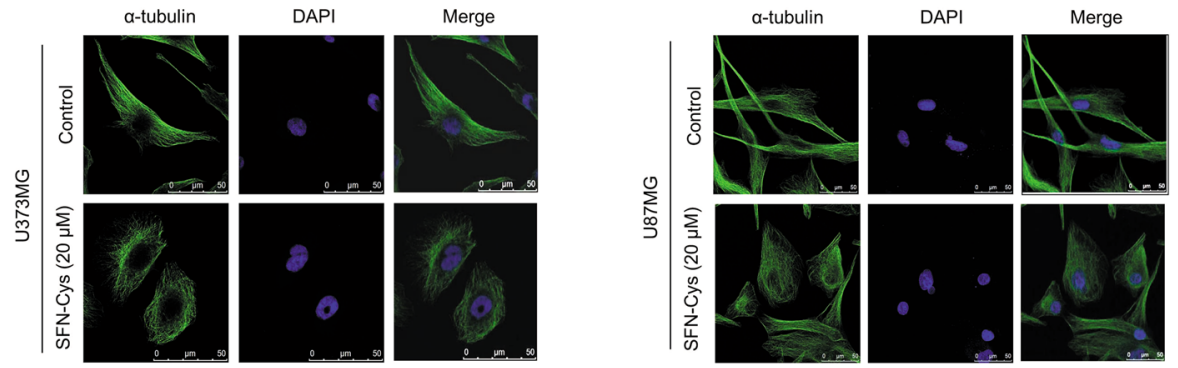

Fig. 3 SFN-Cys changed microtubule morphology and downregulated a-tubulin via sustained activation of ERK1/2. The expression of atubulin was detected by western blot with the treatment of 0, 10, 20, and $30 \mu \mathrm{M} \mathrm{SFN}$-Cys for $24 \mathrm{~h}$ (a) or with the treatment of $20 \mu \mathrm{M}$ SFN-Cys and/or 25 MM PD98059 for $24 \mathrm{~h}$ (b). The expression of Stathmin-1 (c) or pStathmin-1 (Ser 25) (d) was detected by western blot with the treatment of $20 \mu \mathrm{M}$ SFN-Cys and/or $25 \mu \mathrm{M}$ PD98059 for $24 \mathrm{~h}$. e Cells were treated with or without $20 \mu \mathrm{M}$ SFN-Cys and the microtubule morphology was observed by immunofluorescence and confocal microcopy. Scale bar $=50 \mu \mathrm{m}$. $\beta$-actin was used to be the loading control. At least three independent experiments were performed. ${ }^{*} p<0.05$. Data were shown as means $\pm S D(n \geq 3)$.

treated cells by western blot (Fig. 6a, b), suggesting that SFN-Cys downregulated Claudin-5 and S100A4 by inhibiting autophagy. Scratch assay results showed that the relative wound closure $(0 \mathrm{~h}$ vs. $24 \mathrm{~h})$ was elevated in CCCP-treated group indicating mitophagy might promote migration (Fig. 6c), while SFN-Cys treatment decreased the CCCP-upregulated migration in human GBM cells (Fig. 6c). Besides, the relative wound closure was decreased after Baf-A1 treatment (Fig. 6d), while SFN-Cys did not significantly promote Baf-A1- downregulated migration (Fig. 6d). Further invasion assay results also showed that SFN-Cys decreased the invasive cell numbers in GBM (Fig. 6e), while this result was reversed by CCCP (Fig. 6e). Baf-A1 decreased the invasive cell numbers, while this result was exacerbated by SFN-Cys (Fig. 6f). These data indicated that SFN-Cys inhibited cell migration and invasion by repressing mitophagy in GBM cells.

Altogether, we found that SFN-Cys decreased the invasion-associated proteins Claudin-5 and S100A4 


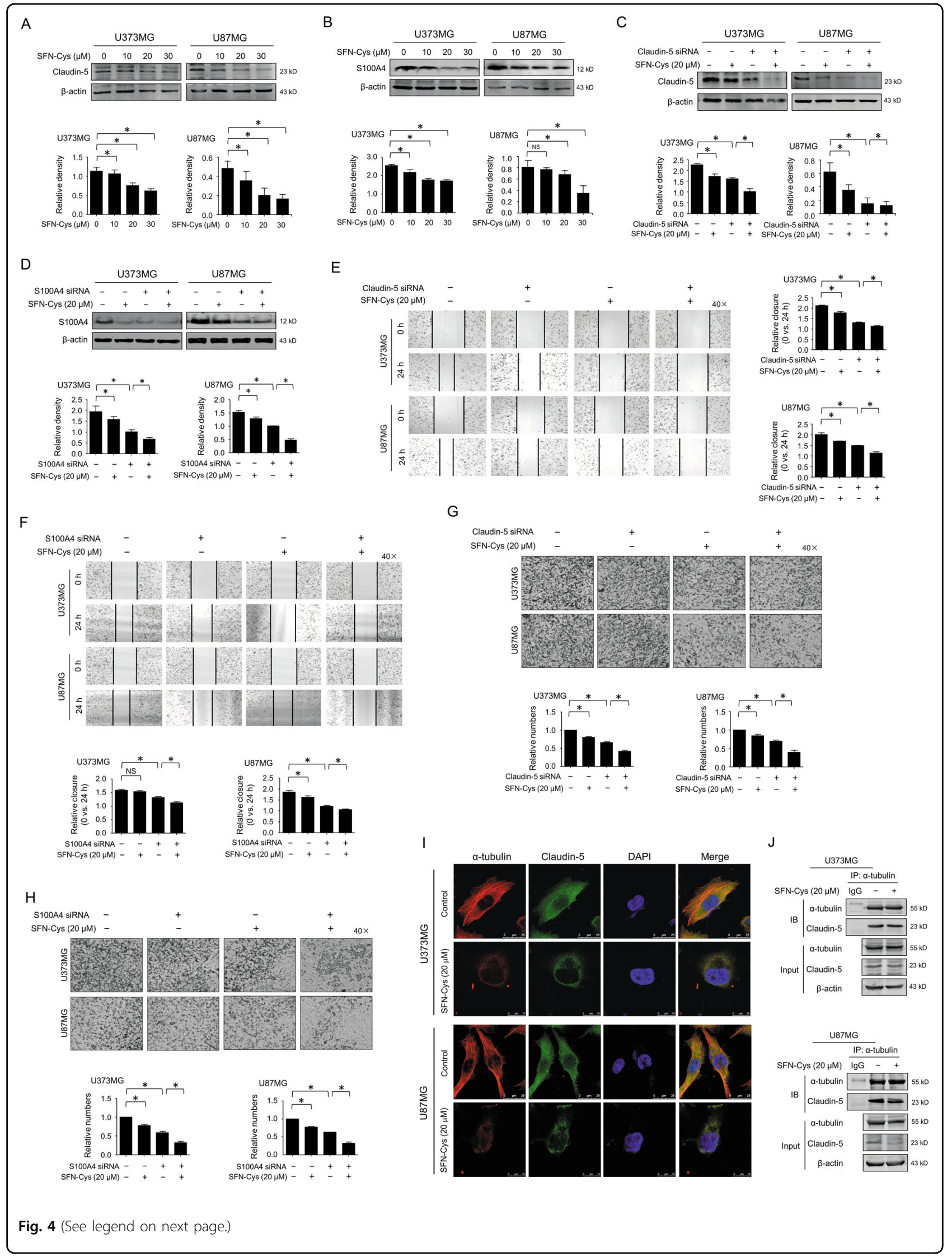


(see figure on previous page)

Fig. 4 SFN-Cys inhibited migration and invasion by downregulating S100A4 and a-tubulin-mediated Claudin-5. The expression of Claudin-5 (a) or S100A4 (b) was detected by western blot with the treatment of 0, 10, 20, and $30 \mu \mathrm{M}$ SFN-Cys for $24 \mathrm{~h}$. The expression of Claudin-5 (c) or S100A4 (d) was detected by western blot with the treatment of 20 MM SFN-Cys and/or knockdown of Claudin-5 (c) or S100A4 (d) for 24 h. Scratch assay was performed to evaluate the migration ability after knockdown of Claudin-5 (e) or S100A4 (f) with or without $20 \mu \mathrm{M}$ SFN-Cys treatment for $24 \mathrm{~h}$. Invasion assay was performed to evaluate the invasion ability after knockdown of Claudin-5 (g) or S100A4 (h) with or without the treatment of $20 \mu \mathrm{M}$ SFN-Cys for $24 \mathrm{~h}$. i Immunofluorescence staining showed the cellular colocalization of Claudin-5 to a-tubulin and the microtubule morphology with the treatment of 20 MM SFN-Cys. Red: stained a-tubulin; green: stained Claudin-5; blue: DAPI-stained DNA; scale bar $=25 \mu \mathrm{m}$. $\mathbf{j}$ Cells were treated with $20 \mu \mathrm{M}$ SFN-Cys for $24 \mathrm{~h}$. The interplay between Claudin- 5 and a-tubulin was detected by co-immunoprecipitation (Co-IP). $\beta$-actin was used to be the loading control. At least three independent experiments were performed. ${ }^{*} p<0.05$; NS no significance. Data were shown as means \pm SD $(n \geq 3)$.

leading to the inhibition of migration and invasion in GBM. Besides, SFN-Cys decreased $\alpha$-tubulin and stathmin-1 by promoting ERK1/2 phosphorylation causing microtubule destruction, the downregulation of mitophagy receptor Bnip3-Nix pathway and the reduction of lysosomal hydrolytic capacity, contributing to the inhibition of mitochondria clearance as well as migration and invasion in GBM cells (Fig. 6g).

\section{Discussion}

In the present study, we demonstrated that SFN-Cys inhibited invasion in GBM cells via inhibiting mitophagy and downregulating invasion-associated protein Claudin5 and S100A4. Invasive process involves multiple signaling pathways; hundreds of proteins might play roles in the cascade. By HPLC-MS/MS analysis we noticed that S100A4 was a target of SFN-Cys in GBM. By GEPIA server, we coincidently found that these GBM patients with higher expression of S100A4 have lower survival. Studies showed that S100A4 was highly expressed in metastatic tumor cells and the expression of S100A4 played a role in regulating GBM cell aggressiveness ${ }^{31}$. S100A4 interacts with cytoskeletal proteins and enhances metastasis of several types of tumor cells ${ }^{32}$. Therefore, we thought that SFN-Cys might downregulate S100A4 and disturb the interaction of S100A4 to microtubules causing the decreasing of invasiveness in GBM. Claudin-5 in endothelial cells promotes vascular permeability and angiogenesis leading to metastasis in tumor cells ${ }^{33}$. Also, Claudin-5 regulates the permeability of blood-brain barrier by modulating the proliferation, migration, and permeability of human brain vascular endothelial cells, especially through the signaling pathway of cell adhe$\operatorname{sion}^{28}$. Bioinformatics analysis showed an increased level of Claudin-5 in GBM tissue type. These indicated that Claudin-5 is also a key invasion-associated biomarker. Further, we uncovered that cell migration and invasion were decreased after knockdown of S100A4 and Claudin5. Here, our results showed that SFN-Cys downregulated the expression of S100A4 and Claudin-5 via phosphorylated ERK1/2. Therefore, SFN-Cys inhibited migration and invasion by ERK1/2-mediated downregulation of S100A4 and Claudin-5.
Recently, we reported that SFN metabolites induced apoptosis and inhibited migration and invasion via causing microtubule disruption ${ }^{7,13,25}$; here we further determined that SFN-Cys inhibited the invasion in GBM by phosphorylated ERK1/2-mediated downregulation of Stathmin- 1 and $\alpha$-tubulin, and microtubule disruption. Stathmin-1 stimulated microtubule catastrophes leading to promotion of microtubule disassembly, while pStathmin-1 (Ser 25) blocked the role of Stathmin- $1^{34}$. Our results showed that SFN-Cys downregulated Stathmin-1 and induced pStathmin-1 by activating ERK1/ 2 , suggesting that SFN-Cys promoted the polymerization of microtubules. However, the double effects of SFN-Cys on both polymerization (by Stathmin-1) and depolymerization (by $\alpha$-tubulin) of microtubules might result in a dynamic imbalance of microtubule, triggering microtubule disruption, and the inhibition of migration and invasion in $\mathrm{GBM}^{13}$.

Autophagy is identified as protective process that is involved in removing damaged organelles including mitochondria, peroxisomes, and endoplasmic reticulum to maintain the balance of cell normal function ${ }^{35}$. Likewise, autophagy plays an important role in regulating tumor cell motility and metastasis ${ }^{24,36}$. Even, autophagy promotes metastatic tumor recurrence; knockdown of autophagy regulators inhibits GBM migration and invasion ${ }^{24}$. We previously revealed that SFN metabolites inhibited autophagy leading to migration and invasion ${ }^{7}$. Autophagy promotes focal adhesion disassembly and cell motility of metastatic tumor cells through the direct interaction of Paxillin to $\mathrm{LC}^{37}$. Notably, previous reports suggested that SFN-NAC blocked the fusion of autophagosome to lysosome leading to the increased level of LC3II/LC3I, LC3II accumulation, and autophagy inhibition in $\mathrm{NSCLC}^{7,25}$. Likewise, here SFN-Cys upregulated LC3II/LC3I but not change SQSTM1/p62 level in GBM cells, indicating SFNCys did not induce the formation of autophagosome. Then we found that SFN-Cys did not aggravate the upregulation of LC3II/LC3I in the present of Bafilomycin A1, suggesting that SFN-Cys-mediated elevation of LC3II/LC3I was not due to the formation of autophagosomes. Similarly, SFNCys did not aggravate the upregulation of LC3II/LC3I in the present of chloroquine, suggesting SFN-Cys might 

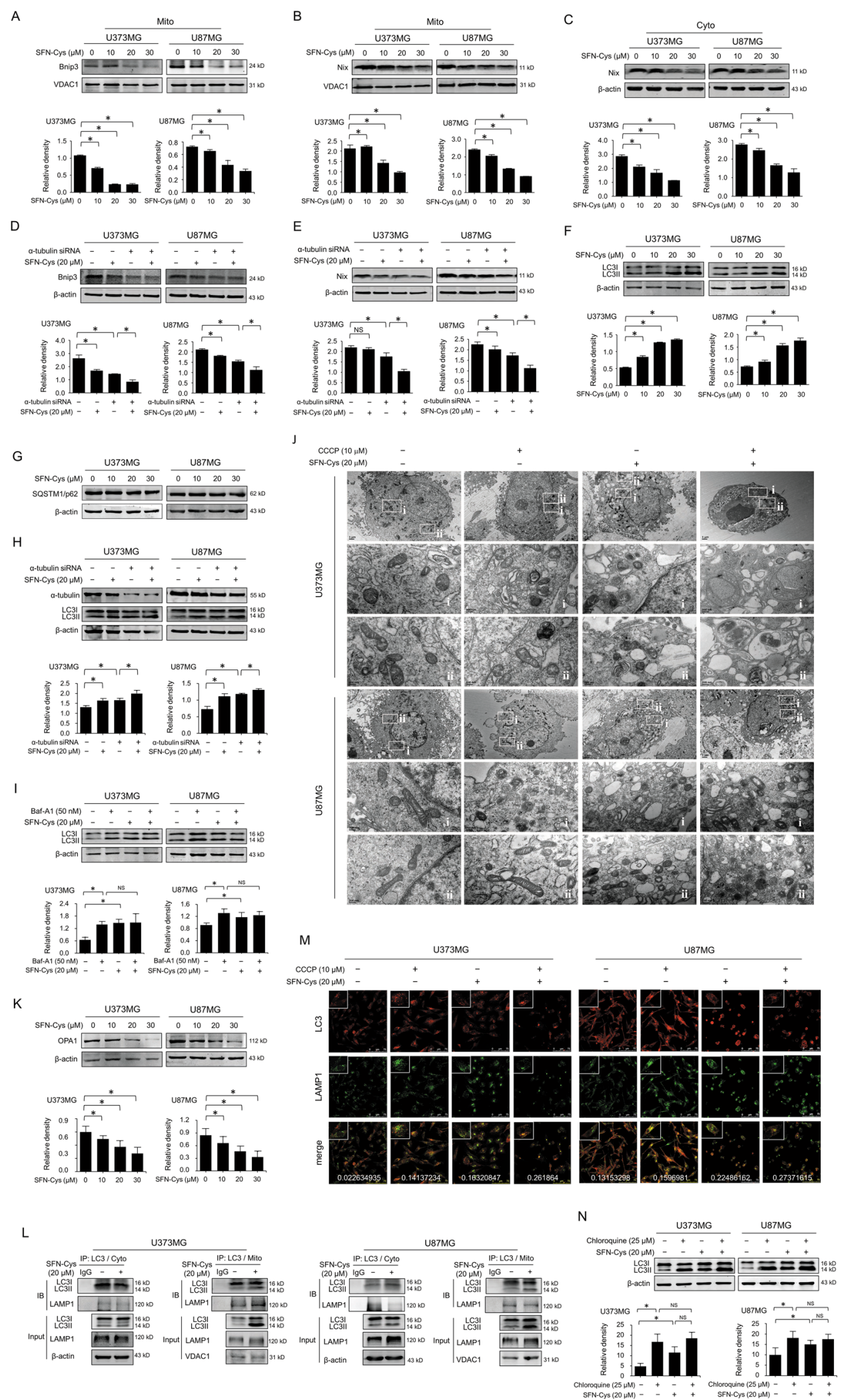

Fig. 5 (See legend on next page.) 
(see figure on previous page)

Fig. 5 SFN-Cys inhibited mitochondrial autophagy by downregulating Bnip3 and Nix and microtubule disruption. The expression of Bnip3 in mitochondria (a) or Nix in mitochondria (b) and in cytosol (c) was detected by western blot with the treatment of 0, 10, 20, and 30 $\mu \mathrm{M}$ SFN-Cys for $24 \mathrm{~h}$. The expression of Bnip3 (d) or Nix (e) was detected by western blot after knockdown of a-tubulin with/without the treatment of $20 \mu \mathrm{M}$ SFN-Cys for $24 \mathrm{~h}$. The expressions of LC3I and LC3II (f) and SQSTM1/p62 (g) were detected by western blot with the treatment of 0, 10, 20, and 30 $\mu$ M SFN-Cys for $24 \mathrm{~h}$. $\mathbf{h}$ The expressions of LC3I and LC3II were detected by western blot after knockdown of a-tubulin with/without the treatment of $20 \mu \mathrm{M}$ SFNCys for $24 \mathrm{~h}$. $\mathbf{i}$ The expressions of LC3I and LC3II were detected by western blot with the treatment of $20 \mu \mathrm{M}$ SFN-Cys and/or $50 \mathrm{nM}$ Bafilomycin A1 for $24 \mathrm{~h}$. $\mathbf{j}$ Subcellular structures in U373MG and U87MG cells were viewed by TEM with $20 \mu \mathrm{M}$ SFN-Cys and/or $10 \mu \mathrm{M}$ CCCP treatment. Scale bar $=1$ and $0.2 \mu \mathrm{m}$ of magnification; " $\mathrm{i}$ " and "ii" in the lower panels matched the two local amplifications of the upper panel in each treated group. $\mathbf{k}$ The expression of OPA1 was detected by western blot with the treatment of $0,10,20$, and $30 \mu \mathrm{M}$ SFN-Cys for $24 \mathrm{~h}$. The interaction of LC 3 to LAMP1 was determined by co-IP both in cytosol and mitochondria treated with $20 \mu \mathrm{M}$ SFN-Cys (I) or by confocal microcopy with the treatment of $20 \mu \mathrm{M}$ SFN-Cys and/or $10 \mu$ M CCCP (m). Red: LC3, green: LAMP1, blue: nuclei. Scale bar $=75 \mu \mathrm{m}$. The local amplification was framed in each image. The colocalization was evaluated by Pearson's_Rr recorded on the merged images. $\mathbf{n}$ The expression of LC3I and LC3II was detected by western blot with the treatment of $20 \mu \mathrm{M}$ SFN-Cys and/or $25 \mu \mathrm{M}$ chloroquine for $24 \mathrm{~h}$. $\beta$-actin and VDAC1 were used to be the loading control of cytosolic/total and mitochondrial proteins. At least three independent experiments were performed. ${ }^{*} p<0.05$; NS no significance. Data were shown as means \pm SD $(n \geq 3)$.

inhibit either the fusion of autophagosomes to lysosomes or lysosomal degradation capacity. Whereas both immunofluorescence staining and co-immunoprecipitation results supported that SFN-Cys enhanced the fusion of autophagosome to lysosome in $\mathrm{GBM}^{38}$. Luckily, we identified that SFN-Cys downregulated both the lysosomal cysteine proteases Cathepsin D and TPP1 in the whole cell and Cathepsin $1 \mathrm{~L}$ in mitochondria by HPLC-MS/MS analysis. Therefore, SFN-Cys might inhibit the hydrolytic capacity and trafficking of lysosomes, blocking the last autophagic flux, impeding autophagosome clearance, and causing the accumulation of autophagosomes in $\mathrm{GBM}^{39}$. Interestingly, these findings revealed a distinct SFN-Cysregulated molecular mechanism in autophagy process compared to NSCLC. In NSCLC, SFN metabolites inhibited autophagic flux by blocking the fusion of mitophagosome to lysosome ${ }^{7,25}$. Studies reported that the expression of several proteins including conjugated LC3, BECN1, and LAMP related to autophagy is increased after injury ${ }^{40}$. Therefore, SFN-Cys-promoted the fusion of autophagosomes to lysosomes might be a feedback for cells against stimuli in GBM. More, we found the LC3II/LC3I was increased after knockdown of $\alpha$-tubulin, suggesting that SFN-Cys might inhibit autophagy via inducing microtubule disruption in GBM. It was reported that autophagy was able to secrete some specific factors, such as interleukin-6, MMP-2, and WNT-5A, which was required for tumor cell invasion $^{41}$. Therefore, based on our studies, it was clear that SFN-Cys promoted microtubule disruption and ultimately inhibited degradation of autolysosomes leading to the inhibition of migration and invasion in GBM.

Generally, hypoxia-induced autophagy is considered nonspecific. Mitophagy is a selective form of autophagy in which mitochondria are specifically targeted for autophagic degradation. Cell motility involves drastic structural changes, a process that demands high levels of energy and fully functional mitochondria whose quality and quantity are balanced by mitophagy ${ }^{42}$. Bnip3 is a proapoptotic atypical $\mathrm{BH} 3$-only protein that has been reported to induce apoptosis, necrosis, or autophagy relying on the type of stress and cellular context ${ }^{43}$. As a hallmark of mitophagy, upregulation of Bnip3 was associated with an increase of autophagy flux and mitochondrial protein degradation, as well as mitophagosome formation; loss of Bnip3 led to the accumulation of damaged mitochondria ${ }^{44}$. Bnip3 contributes to ischemia and reperfusion (I/ $\mathrm{R})$ injury which triggers a protective stress response with upregulation of autophagy and removal of damaged mitochondria ${ }^{45}$. Herein, we found that SFN-Cys decreased mitophagy protein Bnip3 and Nix, and disrupted mitochondria morphology, indicating SFN-Cys might also inhibit mitophagy and hindered the removal of damaged mitochondria. Mitochondria are the critical targets of hypoxia-induced autophagy, and mitochondria dynamics determine the energy supply to reorganize the cytoskeleton and maintain cell movement ${ }^{46}$. Likewise, Bnip3 is also a pro-migratory protein. Deletion of Bnip3 decreases the formation of lamellipodia and filopodia, and the migratory ability of melanoma cells ${ }^{47}$. In our studies, CCCP promoted migration and invasion, whereas Bafilomycin A1 decreased migration and invasion significantly, demonstrating that autophagy/mitophagy was related to migration and invasion. Additionally, Bafilomycin A1 also lowered the expression of invasion-related proteins S100A4 and Claudin-5. Therefore, according to these results, we speculated that the inhibition of autophagy might reduce migration and invasion. In the present study, SFN-Cys downregulated Bnip3 and Nix, inhibiting mitophagy and the clearance of damaged mitochondria, which might result in the inhibition of migration and invasion. Besides, microtubules facilitate autophagosome formation and fusion of autophagosomes to endosomes ${ }^{48}$. Several studies have proposed the direct regulatory effect of mitophagy on cellular migration via stress fibers such as F-actin and tubulin ${ }^{42}$. As we know mitochondrial membrane protein Bnip3 and Nix interact with microtubule lumen protein LC3, which is an important step in the recruitment of mitochondria to nascent autophagosomes, 


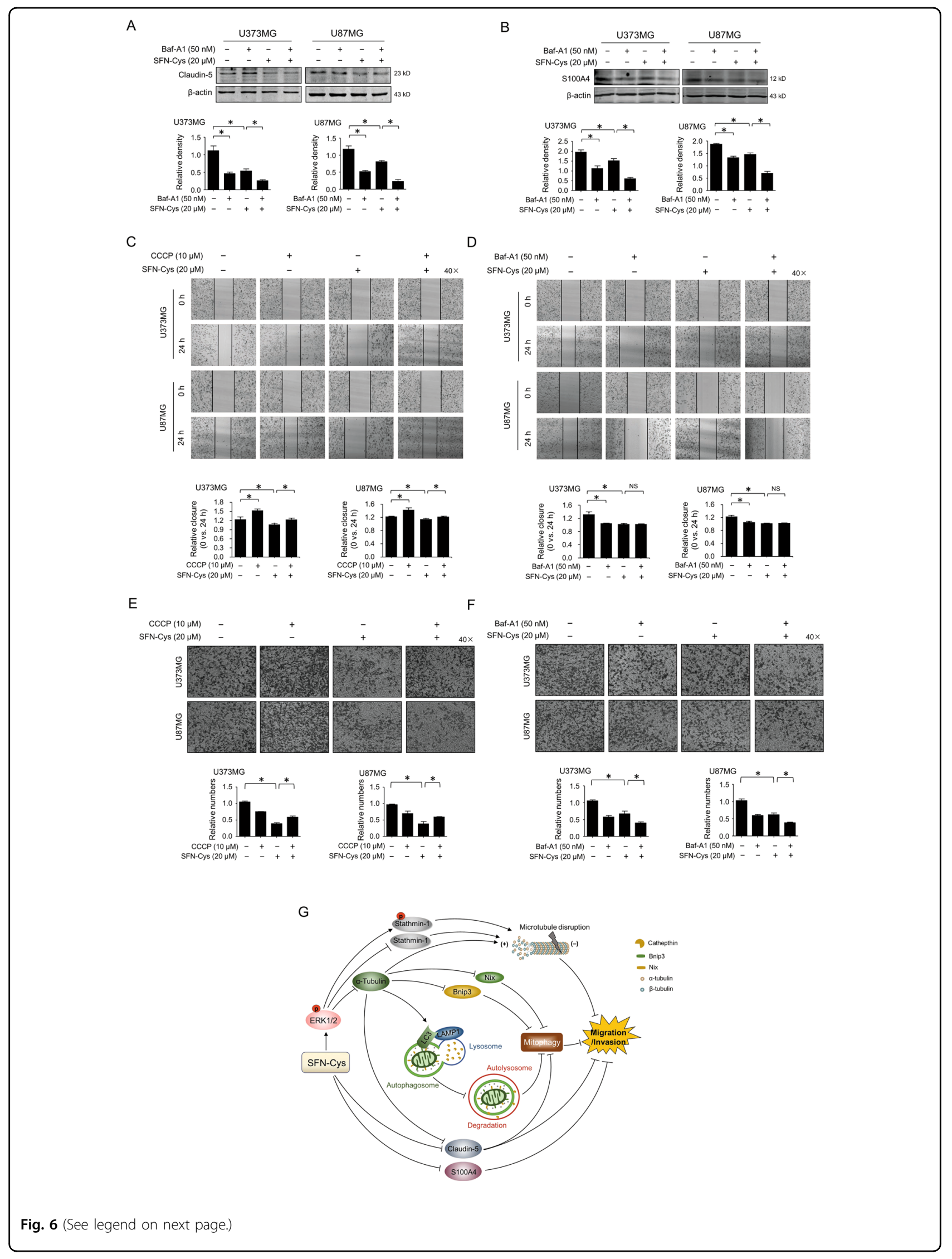


(see figure on previous page)

Fig. 6 SFN-Cys inhibited migration and invasion by inhibiting mitophagy. The expression of Claudin-5 (a) or S100A4 (b) was detected by western blot with the treatment of $20 \mu \mathrm{M}$ SFN-Cys and/or $50 \mathrm{nM}$ Baf-A1 for $24 \mathrm{~h}$. Scratch assay was done to evaluate the migration ability with the treatment with $20 \mu \mathrm{M}$ SFN-Cys and/or $10 \mu \mathrm{M}$ CCCP for $24 \mathrm{~h}$ (c) or with the treatment of $20 \mu \mathrm{M}$ SFN-Cys and/or $50 \mathrm{nM}$ Baf-A 1 for $24 \mathrm{~h}$ (d). The invasion assay was done to evaluate the invasion ability with the treatment of $20 \mu \mathrm{M}$ SFN-Cys and/or $10 \mu \mathrm{M} \mathrm{CCCP} \mathrm{for} 24 \mathrm{~h}$ (e) or with the treatment of $20 \mu \mathrm{M}$ SFN-Cys and/or $50 \mathrm{nM}$ Baf-A1 for $24 \mathrm{~h}$ (f). $\mathbf{g}$ The proposed signaling map for SFN-Cys-inhibited migration and invasion in human glioblastoma. At least three independent experiments were performed. $\beta$-actin was used to be the loading control. ${ }^{*} p<0.05$; NS no significance. Data were shown as means \pm SD $(n \geq 3)$.

indicating microtubule is associated with mitophagy ${ }^{49}$. Additionally, we found that the expression of Bnip3 and Nix was downregulated after knockdown of $\alpha$-tubulin indicating that SFN-Cys triggered microtubule disruption resulting in inhibition of Bnip3/Nix-mediated mitophagy. As such, these outcomes may offer a novel therapeutic strategy against migration and invasion in GBM.

\section{Acknowledgements}

We thank Lei Li at the Facility Center of Capital Medical University for help in HPLC-MS/MS analysis. We thank Zhongxin Xiao at the Facility Center of Capital Medical University for help in immunofluorescence confocal assay. We thank Hui Yang at the Facility Center of Capital Medical University for help in samples preparation for Transmission electron microscope observation. All authors participated in the experiments and reviewed the manuscript. This work was supported by the National Natural Science Foundation of China [grant numbers 81272843, 81601993] and Support Project of High-level Teachers in Beijing Municipal Universities in the Period of 13th Five-Year Plan [grant number IDHT20190510].

\section{Conflict of interest}

The authors declare that they have no conflict of interest.

\section{Publisher's note}

Springer Nature remains neutral with regard to jurisdictional claims in published maps and institutional affiliations.

Supplementary Information accompanies this paper at (https://doi.org/ 10.1038/s41419-020-03024-5).

Received: 29 June 2020 Revised: 13 September 2020 Accepted: 15 September 2020

Published online: 01 October 2020

\section{References}

1. Velásquez, C. et al. Molecular and clinical insights into the invasive capacity of glioblastoma cells. J. Oncol. 2019, 1740763 (2019).

2. Shankar, S., Ganapathy, S. \& Srivastava, R. K. Sulforaphane enhances the therapeutic potential of TRAlL in prostate cancer orthotopic model through regulation of apoptosis, metastasis, and angiogenesis. Clin. Cancer Res. 14, 6855-6866 (2008)

3. Geng, Y. et al. Sulforaphane induced apoptosis via promotion of mitochondrial fusion and ERK1/2-mediated 265 proteasome degradation of novel prosurvival Bim and upregulation of Bax in human non-small cell lung cancer cells. J. Cancer 8, 2456-2470 (2017).

4. Li, C. et al. Sulforaphane inhibits invasion via activating ERK1/2 signaling in human glioblastoma U87MG and U373MG cells. PLoS ONE 9, e90520 (2014).

5. Peng, $X$. et al. Sulforaphane inhibits invasion by phosphorylating ERK $1 / 2$ to regulate E-cadherin and CD44v6 in human prostate cancer DU145 cells. Oncol. Rep. 34, 1565-1572 (2015).

6. Clarke, J. D. et al. Metabolism and tissue distribution of sulforaphane in Nrf2 knockout and wild-type mice. Pharm. Res. 28, 3171-3179 (2011).
7. Zheng, Z. et al. Sulforaphane metabolites inhibit migration and invasion via microtubule-mediated Claudins dysfunction or inhibition of autolysosome formation in human non-small cell lung cancer cells. Cell Death Dis. 10, 259 (2019).

8. Tian, H. et al. Sulforaphane-cysteine suppresses invasion via downregulation of galectin-1 in human prostate cancer DU145 and PC3 cells. Oncol. Rep. 36 1361-1368 (2016).

9. Dogterom, M. \& Koenderink, G. H. Actin-microtubule crosstalk in cell biology. Nat. Rev. Mol. Cell Biol. 20, 38-54 (2019).

10. Hall, A. The cytoskeleton and cancer. Cancer Metastasis Rev. 28, 5-14 (2009).

11. Howell, B., Larsson, N., Gullberg, M. \& Cassimeris, L. Dissociation of the tubulinsequestering and microtubule catastrophe-promoting activities of oncoprotein 18/Stathmin. Mol. Biol. Cell 10, 105-118 (1999).

12. Fife, C. M. et al. Stathmin mediates neuroblastoma metastasis in a tubulinindependent manner via RhoA/ROCK signaling and enhanced transendothelial migration. Oncogene 36, 501-511 (2017).

13. Zhou, Y. et al. Sulforaphane metabolites cause apoptosis via microtubule disruption in cancer. Endocr. Relat. Cancer 25, 255-268 (2018).

14. Escudero-Esparza, A., Jiang, W. G. \& Martin, T. A. Claudin-5 is involved in breast cancer cell motility through the N-WASP and ROCK signalling pathways. J. Exp. Clin. Cancer Res. 31, 43 (2012).

15. Escudero-Esparza, A., Jiang, W. G. \& Martin, T. A. Claudin-5 participates in the regulation of endothelial cell motility. Mol. Cell Biochem. 362, 71-85 (2012).

16. Shimada, H. et al. Downregulation of lipolysis-stimulated lipoprotein receptor promotes cell invasion via claudin-1-mediated matrix metalloproteinases in human endometrial cancer. Oncol. Lett. 14, 6776-6782 (2017).

17. Oku, N., Sasabe, E., Ueta, E., Yamamoto, T. \& Osaki, T. Tight junction protein claudin-1 enhances the invasive activity of oral squamous cell carcinoma cells by promoting cleavage of laminin-5 gamma2 chain via matrix metalloproteinase (MMP)-2 and membrane-type MMP-1. Cancer Res. 66, 5251-5257 (2006).

18. Olinger, E., Houillier, P. \& Devuyst, O. Claudins: a tale of interactions in the thick ascending limb. Kidney Int. 93, 535-537 (2018).

19. $L i, H$. et al. Inactivation of the tight junction gene CLDN11 by aberrant hypermethylation modulates tubulins polymerization and promotes cell migration in nasopharyngeal carcinoma. J. Exp. Clin. Cancer Res. 37, 102 (2018).

20. Yang, D., Du, G., Xu, A., Xi, X. \& Li, D. Expression of miR-149-3p inhibits proliferation, migration, and invasion of bladder cancer by targeting S100A4. Am. J. Cancer Res. 11, 2209-2219 (2017).

21. Chow, K. H. et al. S100A4 is a biomarker and regulator of glioma stem cells that is critical for mesenchymal transition in glioblastoma. Cancer Res. 77, 5360-5373 (2017).

22. Alifieris, C. \& Trafalis, D. T. Glioblastoma multiforme: pathogenesis and treatment. Pharmacol. Ther. 152, 63-82 (2015).

23. Fass, E., Shvets, E., Degani, I., Hirschberg, K. \& Elazar, Z. Microtubules support production of starvation-induced autophagosomes but not their targeting and fusion with lysosomes. J. Biol. Chem. 281, 36303-36316 (2006).

24. Galavotti, S. et al. The autophagy-associated factors DRAM1 and p62 regulate cell migration and invasion in glioblastoma stem cells. Oncogene 32, 699-712 (2013).

25. $\mathrm{Hu}, \mathrm{Y}$. et al. Sulforaphane-N-Acetyl-Cysteine inhibited autophagy leading to apoptosis via Hsp70-mediated microtubule disruption. Cancer lett. 431, 85-95 (2018).

26. Zhang, J. \& Ney, P. A. Role of BNIP3 and NIX in cell death, autophagy, and mitophagy. Cell Death Differ. 16, 939-946 (2009).

27. Chourasia, A. H. et al. Mitophagy defects arising from BNip3 loss promote mammary tumor progression to metastasis. EMBO Rep. 16, 1145-1163 (2015). 
28. Ma, S. et al. Claudin-5 regulates blood-brain barrier permeability by modifying brain microvascular endothelial cell proliferation, migration, and adhesion to prevent lung cancer metastasis. CNS Neurosci. Ther. 23, 947-960 (2017).

29. Tang, Z. et al. GEPIA: a web server for cancer and normal gene expression profiling and interactive analyses. Nucleic Acids Res. 45, W98-W102 (2017)

30. Szklarczyk, D. et al. The STRING database in 2017: quality-controlled proteinprotein association networks, made broadly accessible. Nucleic Acids Res. 45, D362-D368 (2017)

31. Zhang, K., Yu, M., Hao, F., Dong, A. \& Chen, D. Knockdown of S100A4 blocks growth and metastasis of anaplastic thyroid cancer cells in vitro and in vivo. Cancer Biomark. 17, 281-291 (2016).

32. Boye, K. \& Maelandsmo, G. M. S100A4 and metastasis: a small actor playing many roles. Am. J. Pathol. 176, 528-535 (2010).

33. Zeng, Z. et al. Cancer-derived exosomal miR-25-3p promotes pre-metastatic niche formation by inducing vascular permeability and angiogenesis. Nat. Commun. 9, 5395 (2018).

34. Jackson, S. J. \& Singletary, K. W. Sulforaphane: a naturally occurring mammary carcinoma mitotic inhibitor, which disrupts tubulin polymerization. Carcinogenesis 25, 219-227 (2004).

35. Levine, B. \& Klionsky, D. J. Development by self-digestion: molecular mechanisms and biological functions of autophagy. Dev. Cell 6, 463-477 (2004).

36. Lock, R., Kenific, C. M., Leidal, A. M., Salas, E. \& Debnath, J. Autophagydependent production of secreted factors facilitates oncogenic RAS-driven invasion. Cancer Discov. 4, 466-479 (2014).

37. Sharifi, M. N. et al. Autophagy promotes focal adhesion disassembly and cell motility of metastatic tumor cells through the direct interaction of Paxillin with LC3. Cell Rep. 15, 1660-1672 (2016).
38. Wang, J. et al. Silica nanoparticles induce autophagosome accumulation via activation of the EIF2AK3 and ATF6 UPR pathways in hepatocytes. Autophagy 14, 1185-1200 (2018)

39. Bampton, E. T., Goemans, C. G., Niranjan, D., Mizushima, N. \& Tolkovsky, A. M The dynamics of autophagy visualized in live cells: from autophagosome formation to fusion with endo/lysosomes. Autophagy 1, 23-36 (2005).

40. Hagberg, H., Mallard, C., Rousset, C. I. \& Thornton, C. Mitochondria: hub of injury responses in the developing brain. Lancet Neurol. 13, 217-232 (2014).

41. Mowers, E. E., Sharifi, M. N. \& Macleod, K. F. Autophagy in cancer metastasis. Oncogene 36, 1619-1630 (2017).

42. Senft, D. \& Ronai, Z. A. Regulators of mitochondrial dynamics in cancer. Curr Opin. Cell Biol. 39, 43-52 (2016).

43. Zhang, J. et al. Involvement of autophagy in hypoxia-BNIP3 signaling to promote epidermal keratinocyte migration. Cell Death Dis. 10, 234 (2019).

44. Tang, C. et al. Activation of BNIP3-mediated mitophagy protects against renal ischemia-reperfusion injury. Cell Death Dis. 10, 677 (2019).

45. Hamacher-Brady, A. et al. Response to myocardial ischemia/reperfusion injury involves Bnip3 and autophagy. Cell Death Differ. 14, 146-157 (2007).

46. Gugnoni, M., Sancisi, V., Manzotti, G., Gandolfi, G. \& Ciarrocchi, A. Autophagy and epithelial-mesenchymal transition: an intricate interplay in cancer. Cell Death Dis. 7, e2520 (2016).

47. Maes, $H$. et al. BNIP3 supports melanoma cell migration and vasculogenic mimicry by orchestrating the actin cytoskeleton. Cell Death Dis. 5, e1127 (2014).

48. Köchl, R., Hu, X. W., Chan, E. Y. \& Tooze, S. A. Microtubules facilitate autophagosome formation and fusion of autophagosomes with endosomes. Traffic 7, 129-145 (2006)

49. Ashrafi, G. \& Schwarz, T. L. The pathways of mitophagy for quality control and clearance of mitochondria. Cell Death Differ. 20, 31-42 (2013). 